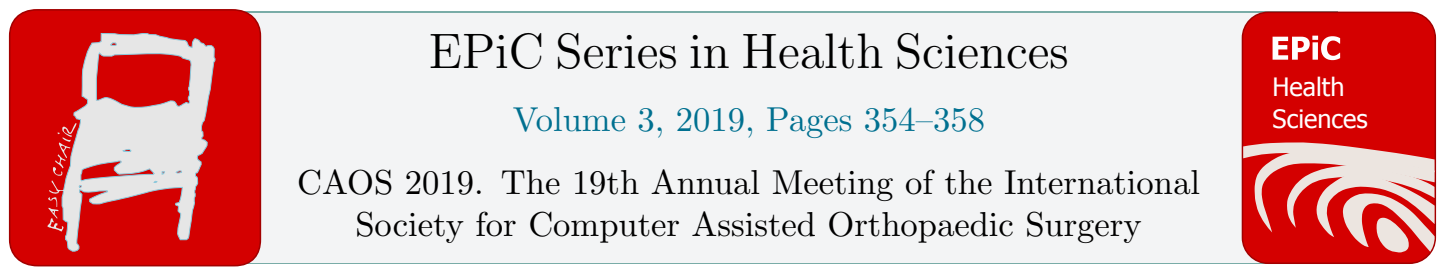

\title{
Comparison effect of Anatomical and Conventional tibial cut in post-operative radiographic and clinical outcome of ankle joint in Computer assisted TKA
}

\author{
Pornpavit Sriphirom ${ }^{1}$, Takrit Chompusaeng ${ }^{2}$, and Bordin Boonton ${ }^{3}$ \\ ${ }^{1}$ Rajavithi Hospital, Rangsit University, Bangkok, Thailand \\ ${ }^{2}$ Rajavithi Hospital, Rangsit University, Bangkok, Thailand \\ ${ }^{3}$ Rajavithi Hospital, Rangsit University, Bangkok, Thailand \\ pornpavit@yahoo.com, TK ortho@hotmail.com, \\ Bordin_boonton@hotmail.com
}

\begin{abstract}
In Total knee arthroplasty (TKA), not only knee joint affect when we restored limb alignment, but also other joints involved. Ankle joint is one of them. By changing in talar tilt, many studies shown this will lead to increase pain and disturb function of ankle. Patients may end up with unsatisfied result of TKA. Anatomical cut of tibia is use in order to avoid this problem.

To compare between pre- and post-operative talar tilt angle (TTA) and ankle clinical assessment by foot function index (FFI) and ankle-hindfoot index (AHI) after anatomical tibial cut (varus $3^{\circ}$ with anatomical axis) and conventional tibial cut (perpendicular with anatomical axis) in computer-assisted total knee arthroplasty

This study was retrospective study. We collected data of 70 knees from 54 patients. 32 knees were done by anatomical cut. The rest were conventional cut. We evaluated at pre- and post-operative for TTA. At 12-month follow-up for TTA \& ankle clinical assessment.

In conventional group, TTA was changed $2.33^{\circ}(\mathrm{p}<0.001)$ then $0.920(\mathrm{p}=0.02)$ at follow up. In anatomical group, TTA was changed $2^{\circ}(\mathrm{p}=0.037)$ then $1.38^{\circ}(\mathrm{p}=1.56)$. Changing of TTA was not significantly different at post-operatively $(\mathrm{p}=0.76)$ and follow-up $(\mathrm{p}=0.98)$ between 2 groups. FFI\& AHI were not significantly different between both groups.
\end{abstract}


Ankle joint always involved when restored alignment of knee that underwent TKA. No matter method of tibial cut was use. But this effect will get better over time and not effect to clinical outcome of ankle.

\section{Introduction}

Goal of total knee arthroplasty (TKA) is painless functional knee with longevity. To get all of these, limb alignment should be restored and soft tissue should be balanced. Beside that surgeons expect patients to satisfy with an operation. In fact, not all results reach to patients' expectation. One study showed up to $20 \%$ of patients who underwent TKA unsatisfied with result ${ }^{1}$. No exact causes were discovered. Some surgeons suspect about alignment changing may play a role in this problem. After correct deformity with conventional tibial cut, not only knee joint affected in changing of alignment, but also other joints involved ${ }^{2-4}$. Ankle joint is one of them. By changing in talar tilt, many studies shown this will result in ankle osteoarthritis, pain and disturb function of ankle ${ }^{2,3}$. These could lead to dissatisfaction of patients. To solve this problem, anatomical tibial cut was proposed. We hope that if we cut tibia about $3^{\circ}$ of varus with anatomical axis, this will be more restore native ankle joint line and better clinical outcome of ankle joint when compared with conventional tibial cut.

\section{Material and methods}

This study was retrospective study. We collected data of 70 knees from 54 patients who underwent CAS-TKA between Jan 2012-Dec 2013 with at least follow up 12 months. All patients are varus OA knee. 32 knees were done by anatomical tibial cut. The rest were conventional tibial cut. Every case was done by single surgeon. Only posterior stabilized design knee (PS) of one company (VEGA knee system, Aesculap ${ }^{\circledR}$ ). CAS-TKA software was OrthoPilot ${ }^{\circledR}$, Aesculap ${ }^{\circledR}$.

We evaluated TTA from standing scanogram at pre-operative, post-operative and 12month follow-up. We measured this angle by 2 residents who do not participate in this study then we used mean of this scores. For ankle clinical assessment, we collected at 12monthfollow-up by phone call.

Data were analyzed by SPFF version 25.0. TTA, FFI and AHI were reported by mean and SD. Post- operative TTA between 2 techniques of tibial cut were analyzed by paired t-test. A difference was statistical significance when p-value is less than 0.05 .

\section{Result}

Patients were 48 (64 knees) women. 16 patients had undergone bilateral TKA. Deformity of OA knee varying about $2-27^{\circ}$ (mean $10.06^{\circ}$ ) in conventional group and $1-26^{\circ}$ (mean $\left.11.1^{\circ}\right)$ in anatomical group. Patient mean age was 69.05 years.

In conventional group, post-operative TTA was $2.7^{\circ}$ (SD 1.64) that changed $2.33^{\circ}$ $(\mathrm{p}<0.001)$ from pre-operative but when 12 -month follow-up TTA reduced to $0.92^{\circ}(\mathrm{p}=0.02)$. In 
anatomical group, post- operative TTA was $2.89^{\circ}(\mathrm{SD} 2.57)$ that changed $2^{\circ}(\mathrm{p}=0.037)$ from preoperative but when 12-month follow-up TTA reduced to $1.38^{\circ}(\mathrm{p}=1.56)$. When compare between both techniques, changing of TTA was not significantly different at post-operatively $(\mathrm{p}=0.76)$ and 12-months follow-up ( $\mathrm{p}=0.98)$.

Ankle clinical assessment, FFI was $35.6 \%$ in anatomical group and $34.4 \%$ in conventional group that not significant difference between group ( $\mathrm{p}=0.80$ ). AHI was $72.67 \%$ in anatomical group and $74 \%$ in conventional group that not significant difference $(\mathrm{p}=0.87)$, too.

\section{Discussion}

From Bourne RB et al, they reported that only $80 \%$ of patients who underwent TKA will be satisfy ${ }^{1}$. To make this rate increase, cause need to find out. Alignment changing of joint may be one of culprits for this problem. Previous studies confirmed that after we restored knee alignment, ankle and foot alignment were affected, too ${ }^{2-4}$. Moreover, Kim reported that changing of ankle joint alignment can aggravate pain in patients underwent TKA ${ }^{3}$.

We assumed that in conventional tibial cut, this will alter the native joint line both knee and ankle. We decided to use anatomical tibial cut to solve this problem. In fact, anatomical tibial cut is hard to reproducible result, so we used CAS-TKA for improve accurate of tibial cutting 5 .

The result of this study shows that no matter what method we used to cut tibia, it always makes ankle joint involved. Same as previous studies of $\mathrm{Kim}^{3}$ and Chompoosang ${ }^{4}$. Difference between group was not statistical significance. When we follow up at 12 months, we found that improvement in radiographic in both groups. We think this happen because adaptability of soft tissue around ankle to concordant with new joint line of knee. This change make clinical parameter of both groups were not different, too.

This finding confirms results of previous studies that alignment of knee will affect to ankle joint.

However, this changing was only little effect and get better over time. Besides, this changing was not affect to clinical function of ankle.

Ankle joint always involved when restored alignment of knee that underwent TKA. No matter method of tibial cut was use. But this effect will get better over time and not effect to clinical outcome of ankle. 
Comparison effect of Anatomical and Conventional tibial cut in post-operative ... P. Sripirom et al.

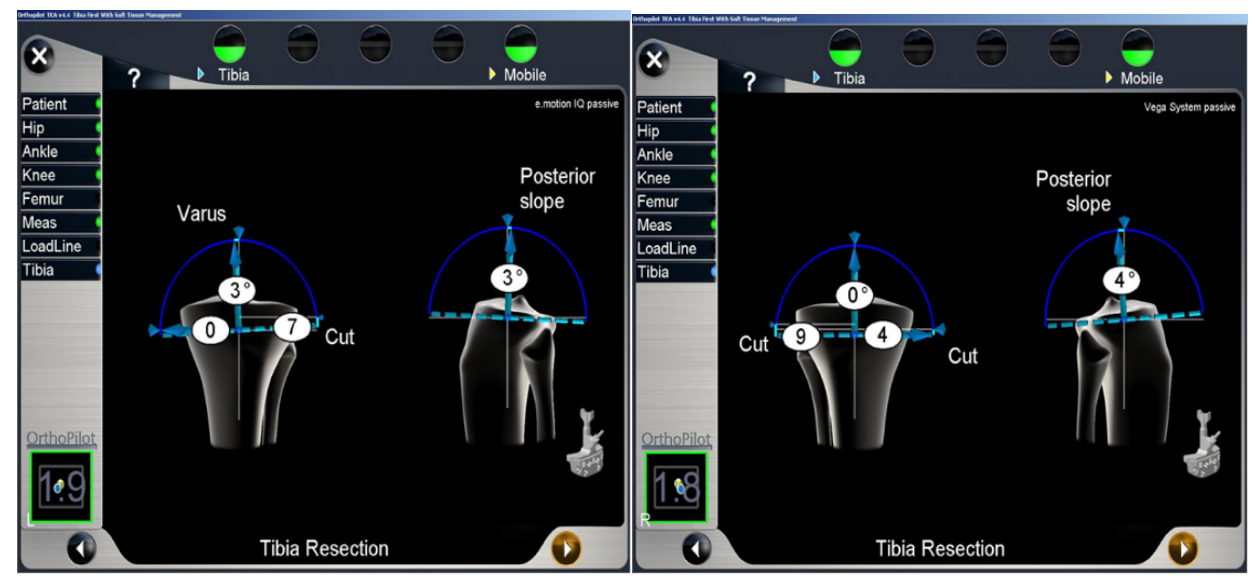

Figure 1 show anatomical tibial cut and conventional tibial cut

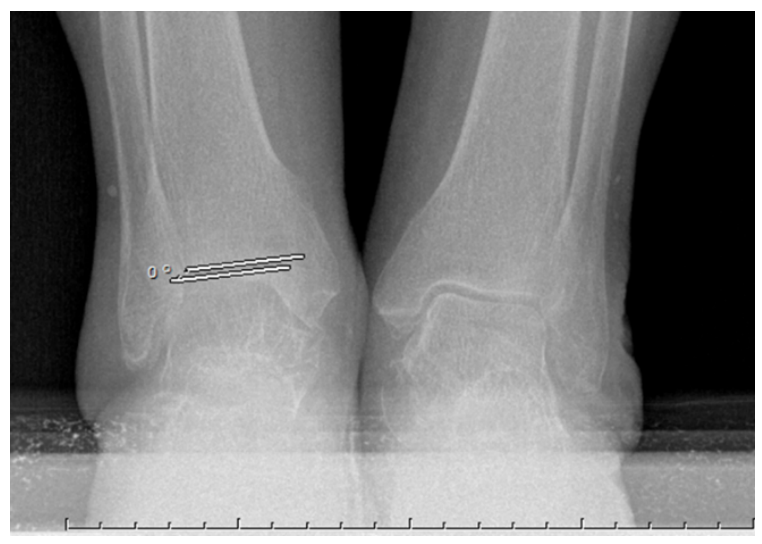

Figure 2 show measure talar tilt angle

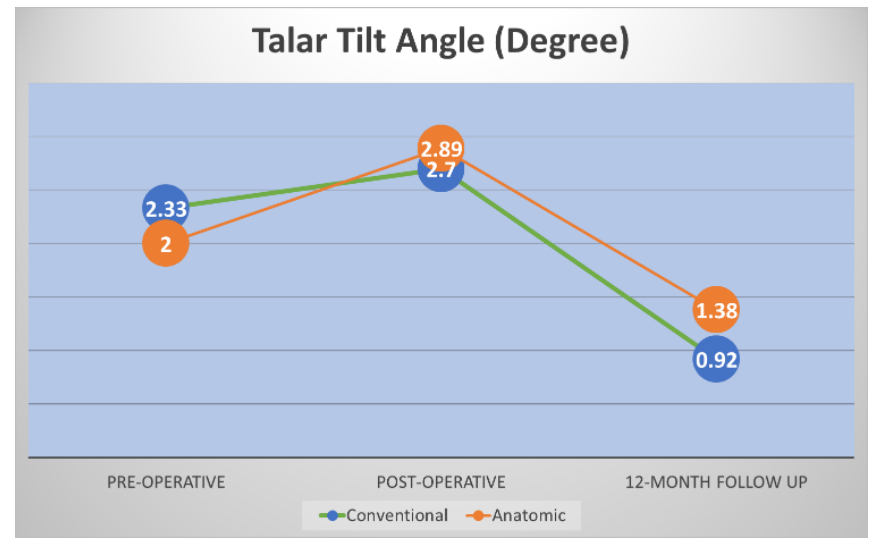

Figure 3 show changing of talar tilt angle both groups 


\section{Reference}

1. Bourne RB, Chesworth BM, Davis AM, Mahomed NN, Charron KDJ: Patient satisfaction after total knee arthroplasty who is satisfied and who is not? Clin Orthop Relat Res 2010;468:57-63.

2. Gwak HC. Change of ankle pain after total knee replacement arthroplasty. J Foot Ankle Res. 2014;7(Suppl 1):A134. Published 2014 Apr 8. doi:10.1186/1757-1146-7S1-A134

3. Kim CW, Gwak HC, Kim JH. Radiologic Factors Affecting Ankle Pain Before and After Total Knee Arthroplasty for the Varus Osteoarthritic Knee. J Foot Ankle Surg. 2018 Sep - Oct;57(5):865-869. doi: 10.1053/j.jfas.2018.02.002. Epub 2018 May 18.

4. Chompoosang T, Khongphaophong M, Chunsiri P, Sripirom P. Computer-Assisted Total Knee Arthroplasty vs. Conventional Total Knee Arthroplasty: Post-Operative Ankle Radiographic Findings and Ankle Clinical Assessment. J Med Assoc Thai. 2016 Feb;99 Suppl 2:S168-74.

5. Jones CW, Jerabek SA. Current Role of Computer Navigation in Total Knee Arthroplasty.J Arthroplasty. 2018 Jul;33(7):1989-1993. doi:

10.1016/j.arth.2018.01.027. Epub 2018 Jan 31. 